\title{
DESIGNS FOR THE COMPLEMENTARY USE OF SYSTEM DYNAMICS AND DISCRETE-EVENT SIMULATION
}

\author{
Jennifer Morgan \\ Susan Howick \\ Valerie Belton \\ Department of Management Science \\ University of Strathclyde \\ Glasgow G1 1QE, UNITED KINGDOM
}

\begin{abstract}
Discrete-Event Simulation (DES) and System Dynamics (SD) are popular modeling approaches that have been successfully applied in a wide range of situations for various purposes. The two approaches can be viewed as complementary, and show potential for combination. Examining multimethodology literature allows us to develop a modeling framework that considers possible designs for such a combination. The aim of this paper is to apply, reflect on and develop this framework through an intervention that lends itself to both approaches, and to explore how DES and SD can be combined in practice. Models under development with a radiotherapy center to explore the impact of altering patient treatment regimes in response to the adoption of new, more complex, technology are discussed. The potential to combine DES and SD in a way which is both complementary and synergistic is explored, and this paper reflects on the experience to date with regard to the proposed methodology.
\end{abstract}

\section{INTRODUCTION}

Extant Discrete-Event Simulation (DES) and System Dynamics (SD) are widely applied modeling approaches that have clearly demonstrated their potential to provide benefit when applied to healthcare situations (Taylor \& Lane 1998; Cooper et al. 2007). In addition, the potential for complementary use of the approaches has been discussed within the broad simulation community (Renshaw 1991; Morecroft \& Robinson 2006), and to healthcare (Brailsford et al. 2010). However, how to create complementary insights, and how the approaches may be combined within the OR field, both in theory and in practice, remains a topic up for discussion (Brailsford et al. 2010).

The research described in this paper brings together DES and SD within the context of the multimethodology literature. It acknowledges prior work on: multimodeling (Yilmaz \& Oren 2004); combined simulation (Praehofer \& Schoeppl 2000); multistage modeling (Davis \& Bigelow 1998); and hybrid modeling (Shanthikumar 1983). Examining the multimethodology literature reveals numerous designs for combining modeling approaches in theory (specifically: Bennett 1985; Schultz \& Hatch 1996; Mingers \& Brocklesby 1997) and allows for consideration of their applicability in practice to the combination of SD and DES. The current research adds to the literature by providing a framework and a coherent set of designs that can be used to inform the development of combined modeling approaches and by examining the application of these designs through a practical project.

The following section will discuss the background to this research including a brief comparison of SD and DES, motivation within the community to utilize combining and the areas where further work is needed. Following this, a framework for methodology selection is proposed and a set of designs for combining modeling approaches is developed from multimethodology literature. Finally, these designs are applied to a case study to explore their potential applicability and relevance. 


\section{BACKGROUND}

SD and DES have been successfully applied to a range of health systems (Brailsford \& Hilton 2000). Both are prevalent yet distinct approaches in the systems modeling field and previous work has highlighted the potential to offer complementary insights with benefits found in combining them; using both prevents becoming "trapped by deterministic fantasy or unnecessary mathematical detail" (Renshaw 1991, p.2).

\subsection{Discrete-Event Simulation}

In DES the dynamics of the system are driven by events. This allows users to model the individual events experienced within a system, with modeling at the entity level to explore progression through a system (Pidd 2004). It can be used to explore a system's ability to meet targets and cope with changes. It is often used to represent systems at an operational level, where the individual detailed interactions and experience of entities over time is important and the variation in service experienced may be a key measure. The model development process of DES is also cyclical, but less so than SD (Tako \& Robinson 2008). Overall, DES has a characteristic style but may be applied in a variety of ways and have different characteristics depending on the problem situation it is applied to. Its application may be described as 'hard' when seeking an accurate representation of a situation (simulation as software engineering) and 'soft' when utilized for problem understanding (Robinson 2002).

\subsection{System Dynamics}

SD is used to discover the underlying principles and behavior of complex systems over time thus capturing the average flow of the system. The efficacy of SD is based on its ability to capture the whole system rather than focusing on short term goals and single measures of performance, which can lead to ineffective conclusions (Taylor \& Dangerfield 2005). It offers a methodology to assist strategy development and policy analysis, capturing information flow and feedback (Sweetser 1999). This is useful within Healthcare to evaluate the long term impact of complex policies (Kuljis 2007). SD models are, in general, a macroscopic view of a system, with an interest in how the system structure impacts the system behavior, recognizing that the behavior of individual components of a system is distinct from the behavior of the system as a whole. The model development processes generally consists of four stages which are cyclical in nature: conceptualization; formulation, testing and implementation, with opportunities for review to reflect on and revisit the stages of the process, referencing the real world throughout (Randers 1980).

\subsection{Comparability and Complementarity}

Randers (1980) presents an early comparison of SD with other forms of quantitative modeling and is discussed by Lane (2000) in relation to DES versus SD. Comparisons of SD specifically with DES are also made by Sweetser (1999), Brailsford and Hilton (2000), Morecroft \& Robinson (2006), Tako \& Robinson (2008) and Chahal \& Eldabi (2008). However, many of these authors seek to place the two approaches into distinct boxes rather than appreciate the similarities. Both approaches are described as providing value and insight to the systems they seek to capture and the problems they aim to address. Sweetser comments that: "Many problems could be modelled by either approach and produce results that would look very similar" (1999, p.8): both tools are suitable for providing increased understanding and aid decisionmaking and in reality, the two approaches demonstrate significant overlap.

All useful simulation models (SD and DES) are simplifications of reality but each approach simplifies differing aspects of a system (Meadows 1980; Pidd 2003). Embedded within each approach are its philosophical assumptions and principles. A primary concern when considering mixing approaches is the issue of paradigm compatibility as both SD and DES have quite separate modeling philosophies (Lane 2000). SD utilizes feedback whilst adopting a system view to examine how causal structure results in observed behavior. DES tends to focus on performance over time, illustrating how randomness influences 
behavior (Tako \& Robinson 2009). However, these differing philosophical views, coupled with capturing a system and its problems at different levels of detail, may yield interesting alternative insights.

Both approaches develop the understanding of a system (Tako \& Robinson 2009). DES often adopts an operational view to understand the detail complexity and explore different configurations (Chahal \&Eldabi 2008). SD may take a strategic view in order to appreciate how a system alters over time (dynamic complexity) and what impact the structure and feedback mechanisms have on the system (Owen 2008). There is no question that each approach has its place, but the primary difference is that DES does not obviously allow the user to understand the underlying mechanics of changing information and feedback; whereas these links and flows are transparent in SD (Tako \& Robinson 2008). Borshchev \& Filippov (2004) discuss that a cross over from DES to SD would be due to the nature of the problem at hand.

This discussion of SD and DES illustrates that it is not possible to simply map and compare the approaches to reveal the potential for combination. It is necessary to obtain an understanding of the situation under study and then explore the potential modeling approaches and whether it is beneficial and appropriate to combine them. The choice between DES and SD "often seems to be made based on an unknown, or at least unstated, user preference function" (Koelling \& Schwandt 2005, p.1322). If SD is efficient in policy design interventions and DES is efficient with policy implementation problems (Ceglowski et al. 2007), the question arises: can we have both? As simulation modeling can be time consuming (An \& Jeng 2005), could a combined approach help to reduce the time taken to produce useful, insightful models or increase the applicability and overall use of models? This discussion leads to the question of when it may be appropriate to combine the approaches, and how they may be used together in a complementary fashion. SD and DES have been combined with, or supported by, other modeling approaches such as statistical analysis, data mining, problem structuring, optimization, Multi Criteria Decision Analysis and process flow mapping. Several papers bringing DES and SD together exist in the literature (examples include: Morecroft \& Robinson 2006; Brailsford et al 2004; Su \& Jin 2008; Helal et al. 2007).

Following the above brief comparison of SD and DES the next section will consider how the two may be formally combined to provide a holistic view of the system, encapsulating the benefits of each method. It will present designs that may be appropriate for bringing the approaches together.

\section{MODELING FRAMEWORKS}

When embarking upon a simulation study two aspects should be examined to decide the focus of the study, and determine the level of accuracy and detail required in the model: the nature of the system (system) and the nature of the study (problem) (Pidd 2004). Merely examining the problem perspective can be misleading (Lane et al. 2000). Further to this, it is important to avoid using the modeling methodology as a starting point for a project, and "select the most suitable methodology for a given purpose and object" (Lorenz \& Jost 2006, p.14).

When learning a modeling approach, a modeler is encouraged to view a system in a certain way and this impacts their choice of tool. Methodology selection is often a personal choice and in practice the modeler can be guided by familiarity with a particular approach (Brailsford \& Hilton 2000). Proponents of a specific approach should take a "step back and assess which conceptual toolkit should be used" (Chick 2006, p.22). Work exploring the model building process of SD and DES empirically supports this commonly held view that modelers will embark on a study without first considering alternative modeling approaches (Tako \& Robinson 2008).

If modelers already have methodology preference, how might we facilitate the selection process to find room for further options to combine approaches? In this paper the authors propose that individual modeler experience and preference plays a significant role, and that a personal filter and appreciation of linking designs needs to sit at the heart of this selection process as outlined in Figure 1. This framework represents the need to examine the system and problem to define the intervention methodology. It also explicitly incorporates the personal filter of modelers that may alter their view of the system and problem. It is suggested that this filter contains bias and so modelers should seek to evaluate alternative options. 


\section{Morgan, Howick and Belton}

Combining modeling approaches raises many philosophical issues which Mingers \& Brocklesby (1997), Mingers et al (1997) and Lane (2000) discuss at length. A major concern for multimethodology is the concept of paradigm incommensurability: the view that paradigms are independent and incompatible as they are based on differing assumptions (Mingers \& Brocklesby 1997). For some it is not conceivable to separate an individual approach from its theoretical backdrop and so when considering mixing approaches it is necessary for the modeler to think at a paradigm level (or indeed at methodology, method or tool/technique level). Cultural and cognitive concerns impact the feasibility of multimethodology studies as organizations and individuals may not be open to the idea of the approach. However, real-world problem situations are highly complex and multidimensional; and potentially may benefit from different paradigms to focus on different aspects of a situation. In the same way that Lane (2000) proposes appreciating both SD and DES to develop a richer understanding; this research proposes that an awareness of mixing would allow us to capitalize on the opportunities it holds.

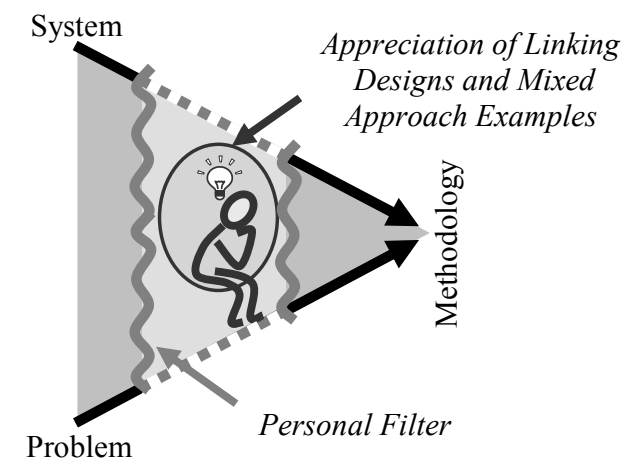

Figure 1: Selection of appropriate modeling approaches

The idea of adopting more than one simulation approach to address a problem situation spans numerous fields such as computer science, engineering, mathematics, and management science. The decidedly "interdisciplinary nature" (Yilmaz \& Oren 2007, p.823) of multilevel and multistage modeling simulation research make it applicable to this work. Multimodeling multiformalism (Zeigler 1977), multisimulation multilevel and multiresolution models, combined simulation (Praehofer \& Schoeppl 2000), and multistage modeling (Davis \& Bigelow 1998) all aim to apply more than one model to develop insight into complex phenomena. Yilmaz \& Oren (2004) propose a taxonomy of multimodels which provides some insight into the structure of the models constructed but does not explicitly consider the design of the intervention to be undertaken and conceptualization concerns.

The term hybrid modeling in MS/OR was first proposed by Shanthikumar (1983) to broadly describe the combination of simulation and analytic models, the combination of which could take several forms. Although it continues to be applicable as an overarching term to describe mixed modeling interventions, it is a vague term that adds little insight into what form the combination may take and how to undertake the modeling process. Examining the multimethodology literature reveals several designs for undertaking multimethodology in theory and their applicability in practice which can in turn inform the combination of SD and DES.

Three key works, which explicitly discuss a range of forms for combining modeling approaches, are:

- Bennett (1985) presents an early discussion of linking methods in which three forms are considered: Comparison, Enrichment and Integration. Comparison is described as a precursor to more ambitious forms of combination, revealing where they are compatible or complementary. Enrichment uses one approach to enhance a second resulting in a single model. Integration involves using elements of existing approaches to create something new.

- Schultz \& Hatch (1996) highlight the importance of the order of combination when combining approaches, by summarizing the possibilities as: Sequential application (where each approach 
remains operating within its paradigm); Parallel (applying approaches on equal terms at the same time; or Interaction (paradigm boundaries are viewed as permeable allowing connections).

- Mingers \& Brocklesby (1997) discuss the overall spectrum of methodology selection, from a single approach (Isolationism) to fully combining two approaches (Multimethodology). Enhancement is proposed (much alike Enrichment) whereby techniques from one methodology are used to enhance another, and combination of whole methodologies are included in this spectrum.

Examination of these key works and their terminology reveals points of commonality. Consequently, a refined set of terms are proposed to eliminate overlap. These terms are proposed for use to inform design when undertaking a study, for reporting purposes, and for reflecting on existing multimethodology:

- Isolationism: Adopting a single approach (including Selection)

- Parallel: Approaches are applied independently and comparisons drawn at fixed points.

- Sequential: One approach follows another.

- Enrichment: A primary approach is enriched with approaches from one or multiple paradigms.

- Interaction: Paradigm boundaries are relaxed allowing connections between approaches.

- Integration: Whole or part approaches are combined to form a new approach. Integration is the proposed term of choice as Multimethodology is often used to refer to the mixed methods field.

This current research continues by examining the use of a 'toolkit' of designs in practice through a healthcare project and takes the following form:

1. Problem \& System Exploration: The principles of the framework to examine the system and the problem are adopted during the project to inform methodology selection and design. A problem structuring approach successfully applied to both SD and DES is employed to reduce bias.

2. Select Issue \& Examine Characteristics: The focus of the project is selected and comparisons of SD and DES are used to identify options for modeling the issues. It is here that consideration is given to the benefit a combined approach might provide over a single methodological approach.

3. Designing Combining: The toolkit of designs is used to support the structuring of the problem and framing of the project. The designs are used to reflect upon how questions may be addressed by the complementary use of SD and DES and how this combination can occur in practice.

4. Model Development: Initial stages of model development are discussed.

The following section discusses a project that is currently in progress which applies the ideas discussed above. The focus of the project is on the radiotherapy processes within an oncology center. The initial problem structuring stages of the process are discussed illustrating the unique roles that SD and DES may take along with their potential complementarity. Preliminary models are presented and the proposed methodology is reflected upon.

\section{PROJECT APPLICATION - THE BEATSON WEST OF SCOTLAND CANCER CENTER}

The Beatson West of Scotland Cancer Centre is Scotland's largest cancer unit serving a population of 2.6 million. The center provides holistic cancer care, but this project is focused specifically on radiotherapy, with the center delivering over 300 doses of radiotherapy per day (www.beatson.scot.nhs.uk/).

Radiotherapy can be used to eradicate cancer cells to eliminate disease (radical treatment) or to relieve cancer symptoms (palliative treatment). Patient treatment is a complex, multistage process that intends to cause as little harm as possible to normal cells by aiming the treatment at the affected area of the body. It requires careful planning and has to be tailored to individual patient physiology. The key stages involved with radiotherapy are booking, simulation, planning, and treatment. These are interrelated stages with feedback throughout as a patients plan may need to be altered, scans may need to be redone or numerous treatment plans revised.

Previous successful projects within the department have examined separately strategic and operational issues with SD and DES respectively. The department continues to face numerous strategic and operational issues, with external and internal influences, and this project provided the client with an opportunity to choose to examine these from a new perspective. 


\subsection{Problem \& System Exploration}

As the problem at the Beatson was not well defined it was necessary to examine potential areas for investigation and determine focus. The initial phase of the project was to explore the system and structure the problem by eliciting issues and views from stakeholders using techniques equally applicable to SD and DES. Causal mapping was utilized to focus on the beliefs, values, and assumptions an individual has about the system and reveal issues in an unbiased manner, enabling the large amounts of information to be collated, and detailed and holistic properties to be explored (Ackermann \& Eden 2005).

Individual interviews were conducted with 7 stakeholders selected to represent a range of views held within the Radiotherapy Department, including management, clinicians and radiotherapy staff. These interviews were semi-structured and aimed to explore the system of interest, highlight aspirations, expose areas of concern, and eventually lead to definition of the problem area(s). Interviews were mapped and merged to form a causal map of the system. This was examined to identify key themes and areas of focus for the project. Interviewees were asked to confirm that the resulting group map of the system reflected their views to ensure relevance. A problem area for initial focus was selected based on both SD and DES type questions raised and discussion.

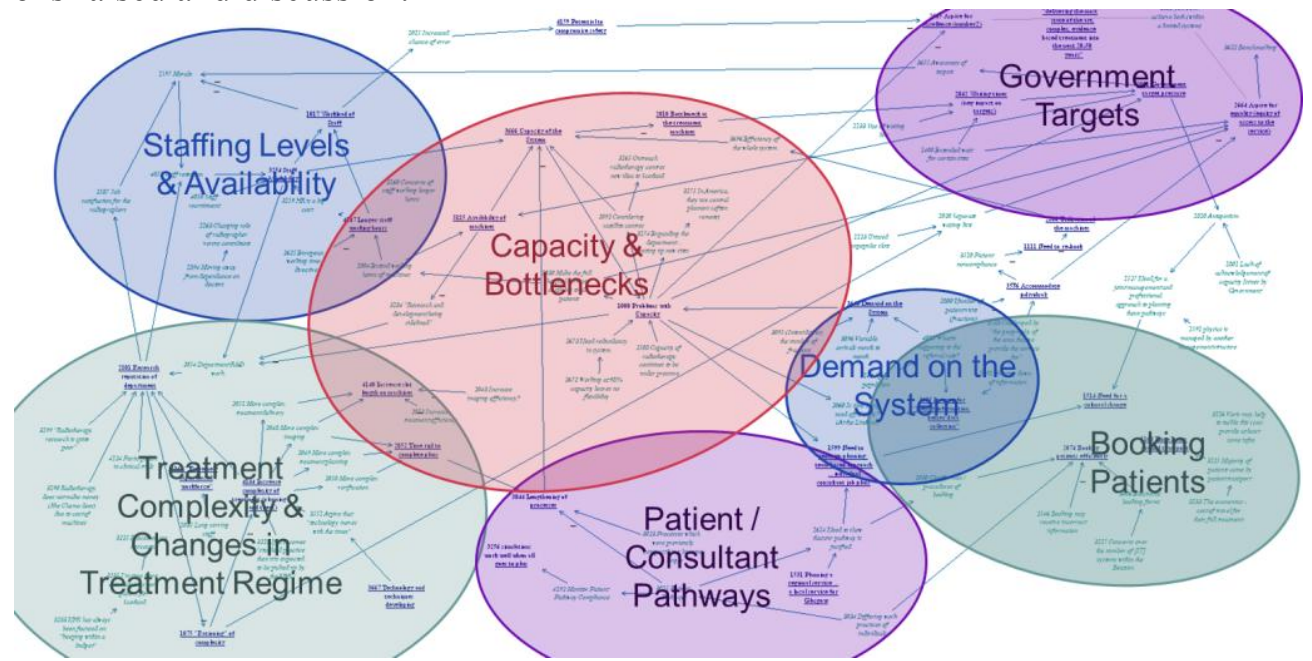

Figure 2: Collective Causal Map. For the purpose of the paper, individual concepts within the cause map are not intended to be readable; rather the aim is to highlight the key themes that emerged.

\subsection{Select Issue \& Examine Characteristics}

Figure 2 shows the collective causal map with surfaced key areas of concern highlighted. This diagram illustrates the interconnectivity and overlap of the key concepts identified within the map. The issue selected for the first study was Treatment Complexity and Changes in Treatment Regime. This problem area was selected due to the immediate nature of the questions being posed (the department is regularly considering implementation of new regimes) and the knock-on impact this aspect has on the capacity of the system and the strain it is put under by increasing workload and requiring new techniques to be learnt.

\subsubsection{Changing Treatment Regimes}

Radiotherapy treatment regimes consist of a plan devised for the treatment of a patient and a series of treatments administered on a linear accelerator. Treatment dosage and the number of fractions (daily visits to the radiotherapy center to receive treatment) are planned. The complexity of these plans can vary for the different cancer types and the characteristics of the patient's disease.

Advances in radiotherapy technology and research, and changing best practices, lead to new techniques becoming available to physicists for the treatment of cancer. The Beatson must decide which treatments to make available to patients and how these can be implemented across the system. Different 


\section{Morgan, Howick and Belton}

developments have differing impacts on the capacity of the system as some may increase efficiency within one part of the system but limit capacity at others.

The nature of the problem is summarized in Figure 3. This diagram has been developed from the collective map and reflects the aspirations of staff (seeking to maintain the Radiotherapy Departments reputation for clinical excellence), whilst ensuring that the impact of implementing new regimes does not negatively impact Key Performance Indicators (KPIs) and equality of access for patients. It also reflects the impact changing radiotherapy regimes has on the capacity of the system by changing the time required imaging, planning and treating patients.

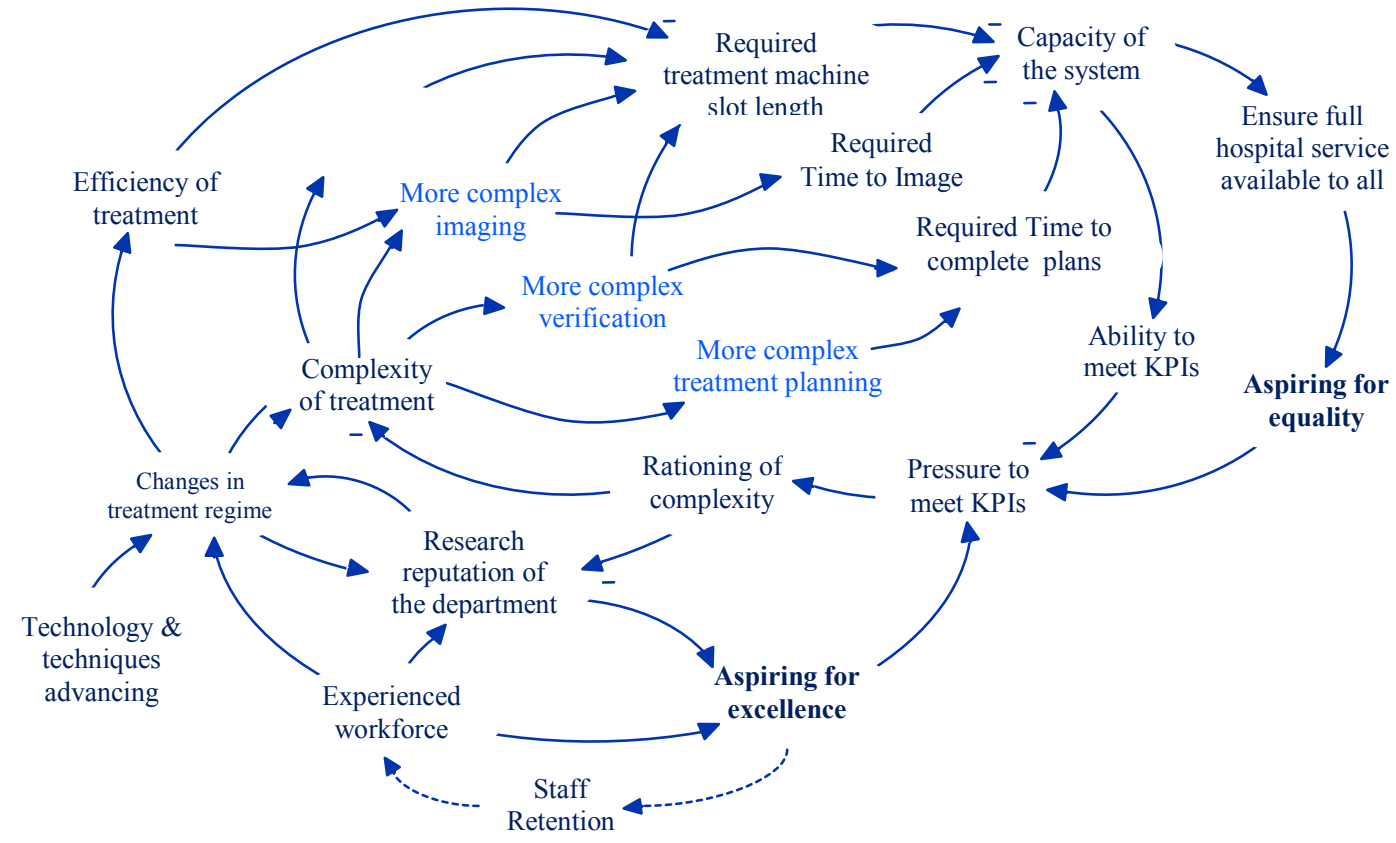

Figure 3: The Problem - Increasing Complexity and Changing Treatment Regimes

\subsubsection{Key Questions}

The maps and interviews were re-examined to determine the key questions being posed around this problem and generate Figure 3 to provide an overview of the causality:

1. What is currently provided at the Beatson and how is availability impacting equality of treatment across all patients? How many patients are currently treated per month, how quickly are patients reaching treatment and how variable is this across patient groups?

2. What is the impact of changing treatment regimes and the mix of regimes? If new regimes are implemented, will it be possible to maintain current throughput; when will the system fail to cope?

3. What is the impact on different patient groups? Will some patients receive a longer wait, will new regimes only become available to cohorts, and will this lead to bottlenecks in the system?

4. What can realistically be implemented and what resources would be required?

5. What would be the impact of a policy change regarding regimes?

These questions display both SD and DES characteristics and a combined approach would allow exploration of both goals, but a single approach would require several assumptions about behavior to be made. In an SD model it would be necessary to assume all patients behave similarly; an SD model is not designed to reveal the range in variability patients may experience within the system. Conversely, within a DES it would be necessary to assume a fixed plan for the implementation of more complex regimes and not allow exploration of the systems propensity to cope with pressure (feedback within the system to reduce complexity). 


\subsection{Designing Combining}

Re-examining the collective map and summarizing the questions above, two key goals become apparent:

Goal A - Explore the dynamics of government targets interacting with R\&D adoption

Goal B - Examine the day to day impact of changing the treatment regime

The proposed design for this project is to use SD to capture the dynamic nature of the problem (Goal A), whilst using DES to appreciate the impact on the day to day running of the center (Goal B). It is felt that the two approaches have explicit roles to play within the modeling intervention and that a complementary approach to modeling be adopted.

In order to examine the day to day impact of changing treatment regimes it is necessary to develop an understanding of the general influence complex technology adoption can have on the system. As discussed in the previous section (modeling frameworks), consideration is given as to how the two approaches can be combined in practice. It was deemed appropriate to adopt a Sequential project design (SD then DES) with the possibility of Interaction once sufficient appreciation of the system was developed and points of exchange identified. SD was selected to be used first to explore the wider system and how government targets and the pressure to maintain low waiting times alter the behavior of the system. This model was then complemented with a DES to examine the individuality of patients' treatment, and the impact altering regimes has on access to the system.

Alternative linking designs, as discussed in Section 3, above, have also been considered. Developing the models in Parallel may result in increased workload as both models take overlapping views of the system. The focus of the SD model remains on the wider system behavior and the DES intends to explore the physical processes within the Beatson. Development of the DES requires significant insight into the system that it was possible to develop whilst constructing the SD model. Enrichment was considered a viable option as stochastic elements of the system could be represented with the SD model. However, this approach would require the inclusion of more individual behavior rather than just the population average (through the use of subscripts) and still may not provide a sufficient level of detail to the client.

This section has outlined the system and problem definition process undertaken with the Beatson. This work led to the focus of the models, the questions to be addressed during the intervention and design of the combination of SD and DES. The following section will outlines the work conducted to date with the Beatson, illustrating the roles each simulation model will take within the project and how understanding gained from the SD model informs the construction of the DES.

\section{MODEL DEVELOPMENT}

\subsection{System Dynamics Model}

A SD model (Figure 4) was developed to capture the dynamics of how the department responds to increasing waiting times by managing working hours and reducing the complexity of the treatments adopted. As more complex treatments are introduced the expected treatment time increases. The initial impact on treatment time is higher than the 'expected' average level due to overall experience within the department dropping and this learning process has an additional impact on the realized treatment time, which impacts the wait experienced by patients. Staff (in the first instance) will try to manage the queue by working longer hours, allowing complexity to be maintained. As waiting times continue to increase the system seeks to reduce the adoption of complexity to balance the system.

This model is used to illustrate the potential management of the system to ensure that waiting times experienced by patients do not exceed government targets (or some other desirable level) by adjusting the pattern of complex treatment adoption and work hours. Delay is built into the model to reflect the time it takes for waiting times to be reported to management and thus to allow for a change in behavior. This model captures the pressure to drive down waiting times, illustrates the careful balance that needs to be maintained within the system and that treatment time needs to be managed carefully. 


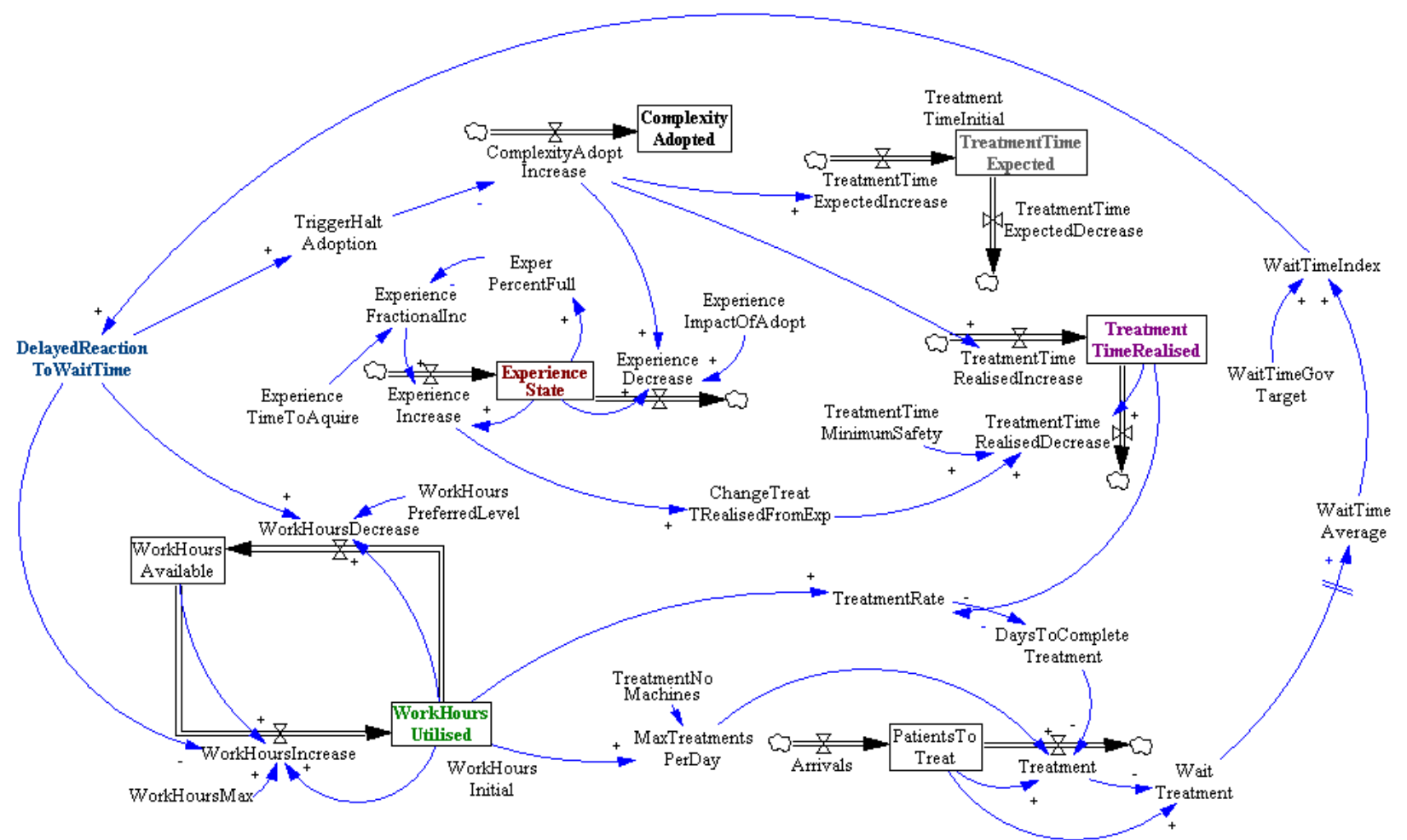

Figure 4: SD Model of the Beatson - treatment complexity impacting waiting times, which influences work hours and complex treatment adoption.

The Beatson treatment process is modeled in the SD model as a single stock and flow, but may be broken down further using a DES to include the intricacies of the flow of patients. The SD model currently represents the average population behavior within the system but further insights can be gained by considering the individualistic behavior of patients and their respective treatment plans. The Beatson has numerous resource restrictions, varying treatment regimes, and a range of routes a patient may take through the treatment process meaning that an increase in treatment complexity can have extensive and diverse impact on the wait time for some cohorts of patients.

\subsection{Discrete-Event Simulation Model}

The DES model (Figure 5) is used to provide more representative insights into the knock-on impact of altering the treatment time or number of treatment fractions for different groups of patients, addressing questions $1,3 \& 4$ from the elicitation process (section 4.4.2 above). Individual patient characteristics, timetables and work plans can be more easily and transparently represented and the DES provides better understanding of the waiting times experienced and the resulting variation across patients. This enables the unit to carefully consider the adoption and implementation of such changes in regime, maintaining the throughput of the system, and minimizing the variation of waiting times experienced by patients, which reflects access to appropriate and timely treatment.

The design of this study is Sequential, using SD to explore the wider context of the problem and develop initial understanding of the concepts involved, followed by DES to consider how to implement changes at ground level. The complementary use of a DES will allow examination of the variation in the impact felt by increasing treatment times or changing the treatment mix. This developed understanding of the knock-on impact can then be used to inform the SD model and the relationship between treatment time and the resulting time a patient takes to progress through treatment (the wait). By examining the 


\section{Morgan, Howick and Belton}

problem through the proposed framework with the view of combining modeling approaches it allows the system to be analyzed at several levels of detail and enables the wider policy issues to be explored.

In the past, the key contact within the Beatson for the project has been drawn to data intensive detailed analysis of the system which is not always practical due to data limitations. The current approach has enabled wider issues to be examined and the general impact of policies on the overall functionality of the system to be assessed before examining the impact at a patient level. It has enabled new questions to be posed and reflections to be made on how the center functions. In addition, the approach has highlighted what measures might be used to assess performance through discussion around the use of throughput versus waiting times versus treatment outcomes.

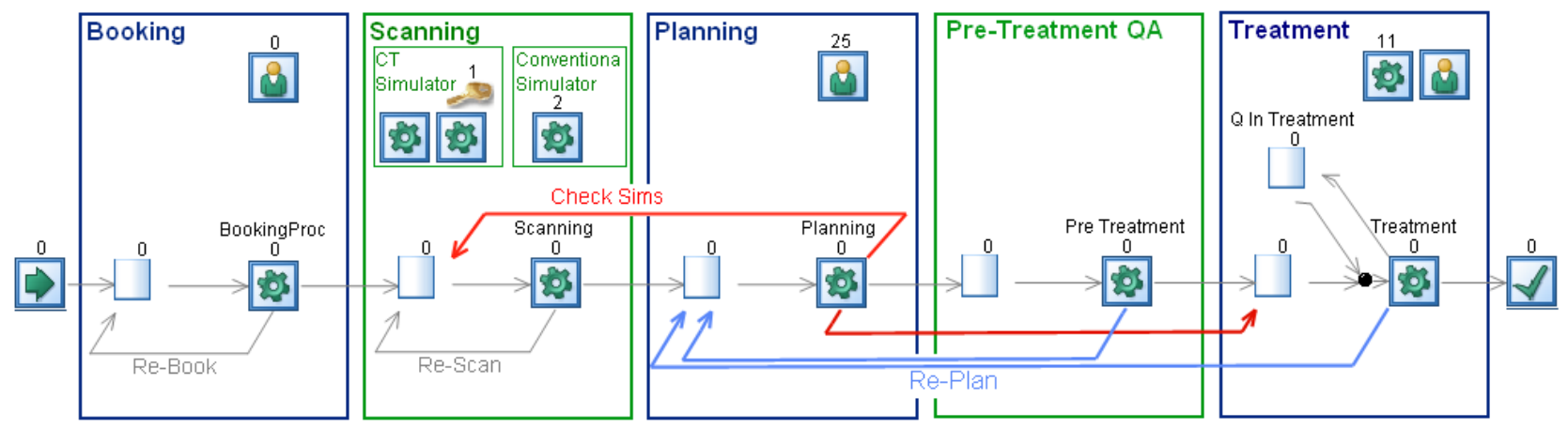

Figure 5: DES Model of the Beatson core process - including booking, scanning, planning, QA and treatment.

\section{DISCUSSION}

For the Beatson project a SD model has been created and could be made more complex to include much of the DES's functionality. However it is believed that this would stretch and contort the SD paradigm. DES is proposed for use to add value to the modeling of the treatment process, and then the two models may be linked. The design of this intervention is sequential (SD then DES) with the aim to have the two models interacting to provide further insights. Although work is still underway, this case study illustrates that linking SD then DES to a problem works in practice and that the problem dictates the linking design adopted. In theory there is a wide range of linking options available to a modeler, but in practice it is necessary to carefully consider the practicalities of the design adopted ensuring that it is the most efficient use of the modeler and the client(s) time and resources. Within the case study it is possible to develop large complex models and apply the range of linking designs available, but these may not fit with the clients' desired outputs, including unnecessary detail.

This paper has discussed how SD and DES may be viewed from several perspectives and applied in a variety of ways depending on the modeler and that it is necessary to evaluate the suitability of mixing approaches on a case by case basis. The purpose of this work has been to explore how the approaches may be used together to provide complementary insight into a situation, and how the two approaches may interact to provide such insight. The linking designs have been used to aid the planning of an effective intervention: to inform the development of the models and their combined use, enable clear discussion of the form the combination may take, and provide a coherent set of designs for implementation. Although this work focused on the potential benefit of mixing SD and DES, the designs may be used to explore the mixing of other modeling approaches. The work therefore contributes to the wider field that reflects the practice and theory of multimethodology.

This paper presents a work in progress, with the DES model currently in development. Further work includes running various scenarios within the models. Experimental results will follow, allowing their overall usefulness and applicability within the context of the case, and their suitability for answering the questions posed, to be assessed and insights to be reflected upon. Feedback from the stakeholders has been positive throughout the project and, following scenario analysis, it will be possible to engage with 


\section{Morgan, Howick and Belton}

the stakeholders and assess their personal views on the process undertaken. This would allow reflection on the simulation methods adopted, how the two methods are perceived, and their usefulness within this case. This would also provide an opportunity to gain insight into how practical and relevant mixed modeling is viewed to be, and the potential usefulness of the overall process undertaken.

\section{ACKNOWLEDGEMENTS}

With thanks to the Beatson for their continuing involvement and support throughout this project; to the EPSRC, Simul8 and the Beatson Fund for sponsorships; and to the reviewers for their valuable feedback.

\section{REFERENCES}

The Beatson West of Scotland Cancer Centre. www.beatson.scot.nhs.uk/ [Last Accessed 03/14/2011].

Ackermann, F. and C. Eden. 2005. "Using Causal Mapping with Group Support Systems to Elicit an Understanding of Failure in Complex Projects: Some Implications for Organisational Research." Group Decision and Negotiation 14:355-376.

An, L. and J. Jeng. 2005. "On Developing System Dynamics Model for Business Process Simulation.” In Proceedings of the 2005 Winter Simulation Conference, edited by M. E. Kuhl, N. M. Steiger, F. B. Armstrong, and J. A. Joines. Piscataway, New Jersey: Institute of Electrical and Electronics Engineers, Inc.

Bennett, P. G. 1985. “On Linking Approaches to Decision Aiding: Issues and Prospects.” J. Op. Res. Soc. 36:659-669.

Borshchev, A. \& Filippov, A. 2004. "From System Dynamics and Discrete Event to Practical Agent Based Modeling: Reasons, Techniques, Tools." Proc. 22nd Int. Conf. System Dynamics Soc. Oxford, England.

Brailsford, S. C., Hilton, N. 2000. "A Comparison of Discrete Event Simulation and System Dynamics for Modelling Healthcare Systems." In Proc. 26th meeting of the ORAHS Working Group. Glasgow, 18-39.

Brailsford, S. C., Lattimer, V. A., Taranas, P. \& Turnbull, J. C. 2004. "Emergency and On-Demand Health Care: Modelling a Large Complex System." J. Op. Res. Soc. 55:34-42.

Brailsford, S. C., Desai, S. M \& Viana, J. 2010. "Towards the Holy Grail: Combining System Dynamics and Discrete Event Simulation in Healthcare." In Proceedings of the 2010 Winter Simulation Conference, edited by B. Johansson, S. Jain, J. Montoya-Torres, J. Hugan, and E. Yücesan, 2293-2303. Piscataway, New Jersey: Institute of Electrical and Electronics Engineers, Inc.

Ceglowski, R., Churilov, L. \& Wasserthiel, J. 2007. "Combining Data Mining and Discrete Event Simulation for a Value-added view of a hospital emergency department." J. Op. Res. Soc. 58:246-254.

Chahal, K. \& Eldabi, T. 2008. "Which is more appropriate: A multiperspective comparison between System Dynamics and Discrete Event Simulation." In Proc. European and Mediterranean Conf. on Information Systems.Dubai.

Chick, S. E. 2006. "Six ways to improve a simulation analysis." J. Simulation. 1:21-28.

Cooper, K., Brailsford, S. C. \& Davies, R. 2007. "Choice of Modelling Technique for Evaluating Healthcare Interventions.” J. Op. Res. Soc. 58:168-176.

Davis, P. K. \& Bigelow, J. H. 1998. "Experiments in Multiresolution Modeling (MRM)." RAND Corporation.

Helal, M., Rabelo, L., Sepulveda, J. \& Jones, A. 2007. “A Methodology for Integrating and Synchronizing the System Dynamics and Discrete Event Simulation Paradigms." Proc. 25th Int. Conf. System Dynamics Soc. Boston.

Koelling, P. \& Schwandt, M. J. 2005. "Health Systems: A Dynamic System - Benefits from System Dynamics." In Proceedings of the 2005 Winter Simulation Conference, edited by M. E. Kuhl, N. M. 
Steiger, F. B. Armstrong, and J. A. Joines. Piscataway, New Jersey: Institute of Electrical and Electronics Engineers, Inc.

Kuljis, J., Paul, R. J. \& Stergioulas, L. K. 2007. Can health care benefit from modelling and simulation methods in the same way as business and manufacturing. In Proceedings of the 2007 Winter Simulation Conference, edited by S. G. Henderson, B. Biller, M.-H Hsieh, J. Shortle, J. D. Tew, and R. R. Barton, 1449-1453. Piscataway, New Jersey: Institute of Electrical and Electronics Engineers, Inc.

Lane, D. C. 2000. "You Just Don't Understand Me: Modes of failure and success in the discourse between system dynamics and discrete event simulation." LSE OR Working Paper 00.34.

Lane, D. C., Monefeldt, C. \& Rosenhead, J. V. 2000. "Looking in the wrong place for healthcare improvements: A system dynamics study of an accident and emergency department." J. Op. Res. Soc. 51:518-531.

Lorenz, T. \& Jost, A. 2006. "Towards an orientation framework in multi-paradigm modeling - Aligning purpose, object and methodology in System Dynamics, Agent-based Modeling and Discrete-EventSimulation." In. Proc. 24th Int. Conf. System Dynamics Soc.

Meadows, D. H. 1980. The Unavoidable A Priori. In: Randers, J. (ed.) Elements of the System Dynamics Method. Waltham, MA: Pegasus Communications.

Mingers, J. \& Brocklesby, J. 1997. "Multimethodology: Towards a Framework for Mixing Methodologies." Omega. 25:489-509.

Mingers, J., Gill, A., Ackermann, F., Bennett, P., et al. 1997. Multimethodology: The Theory and Practice of Combining Management Science Methodologies, John Wiley \& Sons.

Morecroft, J. D. W. \& Robinson, S. 2006. "Comparing Discrete-Event Simulation and System Dynamics: Modelling a fishery.” In: Robinson, S., Taylor, S., Brailsford, S. C. \& Garnett, J., eds. Proc. OR Soc. Simulation Workshop.

Owen, C., Love, D. \& Albores, P. 2008. "Selection of Simulation Tools for Improving Supply Chain Performance." In: Proc. 50th Conf. Op. Res. Soc. 199-207.

Pidd, M. 2003. Tools for Thinking: Modelling in Management Science, John Wiley \& Sons.

Pidd, M. 2004. Computer Simulation in Management Science, John Wiley \& Sons.

Praehofer, H. \& Schoeppl, A. 2000. "A Continuous and Combined Simulation Platform in JAVA and its Application in Building Paper Mill Training Simulators.” In: Proc. of WebSim 2000. San Diego.

Randers, J. 1980. Elements of the System Dynamics Method. Waltham, MA: Pegasus Communications.

Renshaw, E. 1991. Modelling Biological Populations in Space and Time, University of Cambridge.

Robinson, S. 2002. "Modes of simulation practice: approaches to business and military simulation." Simulation Modelling Practice and Theory. 10:513-523.

Schultz, M. \& Hatch, M. J. 1996. "Living with Multiple Paradigms: The Case of Paradigm Interplay in Organisational Culture Studies." Ac. Mgmt Rev. 21:529-557.

Shanthikumar, J. G. \& Sargent, R. G. 1983. "A Unifying View of Hybrid Simulation/Analytic Models and Modeling." Operations research, 31, 1030-1052.

Su, Y. \& Jin, Z. 2008. "Modeling Health Service Centers with Simulation and System Dynamics." In Proc. Tenth Int. Conf. on Computer Modeling and Simulation. 649-654.

Sweetser, A. 1999. "A Comparison of System Dynamics (SD) and Discrete Event Simulation (DES)." In Proc. 17th Int. Conf. of the System Dynamics Soc. Wellington, NZ.

Tako, A. A. \& Robinson, S. 2009. "Comparing discrete-event simulation and system dynamics: users' perceptions.” J. Op. Res. Soc. 60:296-312.

Taylor, K. \& Dangerfield, B. 2005. "Modelling the feedback effects of reconfiguring health services." $J$. Op. Res. Soc. 56:659-675.

Taylor, K. \& Lane, D. C. 1998. "Simulation applied to health services: opportunities for applying the system dynamics approach." J. of Health Services Research and Policy. 3:226-232.

Yilmaz, L. \& Oren, T. I. 2004. "Dynamic Model Updating in Simulation with Multimodels: A Taxonomy and a Generic Agent-Based Architecture." In: Summer Computer Simulation Conf. California. 
Yilmaz, L. \& Oren, T. I. 2007. "Requirements and Design Principles for Multisimulation with Multiresolution, multistage Models." In Proceedings of the 2007 Winter Simulation Conference, edited by S. G. Henderson, B. Biller, M.-H Hsieh, J. Shortle, J. D. Tew, and R. R. Barton, 823-832. Piscataway, New Jersey: Institute of Electrical and Electronics Engineers, Inc.

Zeigler, B. 1977. "Multi-Model, Multi-Formalism Modelling: An Ecosystem Example." In: Halfo, E. (ed.) Theoretical Systems Ecology: Advances and Case Studies. New York: Academic Press.

\section{AUTHOR BIOGRAPHIES}

JENNIFER MORGAN is a final year PhD student in the Department of Management Science at the University of Strathclyde. Jennifer obtained a BSc in Mathematics from Cardiff University and an MSc in Operational Research from Strathclyde. Her PhD research is sponsored by the UK Engineering and Physical Sciences Research Council, Simul8 Corporation and the Beatson Oncology Fund, and she is supervised by Valerie Belton and Susan Howick. She can be contacted at: jennifer.s.morgan@strath.ac.uk

SUSAN HOWICK is a Professor in the Management Science Department at the University of Strathclyde in Glasgow. Susan has a BSc in Mathematics, an MSc in Operational Research and a PhD in Management Science. Her research has involved the use of system dynamics modeling to support decisionmakers in a number of areas; for example in engineering project post-mortems, valuing renewable energy and telecommunications. Susan's research has particularly focused on the added value that can be gained by integrating system dynamics with other modeling methodologies.

VALERIE BELTON is a Professor in the Management Science Department at the University of Strathclyde in Glasgow. Valerie gained degrees in mathematics and OR from Durham and Lancaster Universities respectively, and completed a Doctorate at Cambridge. Her principal focus of research interest has been Multicriteria Decision Analysis (MCDA), with a particular area of interest being effective problem structuring and model building and integrating problem structuring methods, in particular the use of cognitive mapping, with MCDA. 